\title{
TI.2.1
}

\section{The Document Repository Service}

- PDF: TheDocumentRepositoryService.pdf

- Text: TheDocumentRepositoryService.txt

\section{More Information}

\begin{tabular}{|l|l|}
\hline Repository ID & TI.2.1 \\
\hline Persistent URL & http://doi.org/10.26869/TI.2.1 \\
\hline Title & The Document Repository Service \\
\hline Authors & David Walker, Tom Barton, Emily Eisbruch, Ann West, Mike Zawacki \\
\hline Sponsor & Internet2 \\
\hline Review & Document Stewardship Community Review \\
\hline Status & Preserve \\
\hline Publish Date & $11 / 11 / 2016$ \\
\hline DOI & $10.26869 /$ TI.2.1 \\
\hline Signature & \\
\hline Deprecated & No \\
\hline Future Review & $3 / 1 / 2017$ \\
\hline Supersedes & \\
\hline Format & \\
\hline Related Docs & \\
\hline Development Location & \\
\hline IP Framework & CC BY 4.0 \\
\hline Subject Tags & \\
\hline Notes & \\
\hline
\end{tabular}

